\title{
Bronchiolitis obliterans syndrome and restrictive allograft syndrome after lung transplantation: why are there two distinct forms of chronic lung allograft dysfunction?
}

\author{
Masaaki Sato \\ Department of Thoracic Surgery, The University of Tokyo Graduate School of Medicine, Tokyo, Japan \\ Correspondence to: Masaaki Sato, MD, PhD. 7-3-1 Hongo, Bunkyo-ku, Tokyo, 113-8655, Japan. Email: satom-sur@h.u-tokyo.ac.jp.
}

\begin{abstract}
Bronchiolitis obliterans syndrome (BOS) had been considered to be the representative form of chronic rejection or chronic lung allograft dysfunction (CLAD) after lung transplantation. In BOS, small airways are affected by chronic inflammation and obliterative fibrosis, whereas peripheral lung tissue remains relatively intact. However, recognition of another form of CLAD involving multiple tissue compartments in the lung, termed restrictive allograft syndrome (RAS), raised a fundamental question: why there are two phenotypes of CLAD? Increasing clinical and experimental data suggest that RAS may be a prototype of chronic rejection after lung transplantation involving both cellular and antibody-mediated alloimmune responses. Some cases of RAS are also induced by fulminant general inflammation in lung allografts. However, BOS involves alloimmune responses and the airway-centered disease process can be explained by multiple mechanisms such as external alloimmune-independent stimuli (such as infection, aspiration and air pollution), exposure of airway-specific autoantigens and airway ischemia. Localization of immune responses in different anatomical compartments in different phenotypes of CLAD might be associated with lymphoid neogenesis or the de novo formation of lymphoid tissue in lung allografts. Better understanding of distinct mechanisms of BOS and RAS will facilitate the development of effective preventive and therapeutic strategies of CLAD.
\end{abstract}

Keywords: Lung transplantation; bronchiolitis obliterans syndrome (BOS); restrictive allograft syndrome (RAS); chronic rejection

Submitted Feb 06, 2020. Accepted for publication Feb 29, 2020.

doi: $10.21037 / \mathrm{atm} .2020 .02 .159$

View this article at: http://dx.doi.org/10.21037/atm.2020.02.159

\section{Introduction}

Restrictive allograft syndrome (RAS) is an established phenotype of chronic lung allograft dysfunction (CLAD) after lung transplantation (1). Unlike bronchiolitis obliterans syndrome (BOS), which shows obstructive physiology, RAS shows restrictive physiology. Pathologically, BOS is represented by obliterative bronchiolitis (OB) in small airways associated with inflammation and fibrosis, while peripheral lung tissues (such as alveoli and pleura) remain relatively intact (2). Conversely, RAS shows peripheral lung fibrosis with various patterns including pleuroparenchymal fibroelastosis, diffuse alveolar damage, acute fibrinoid organizing pneumonia and organizing pneumonia in addition to OB (1,3-5). Such different microscopic findings are reflected by obviously distinct radiological findings of the two syndromes, which show a similar contrast to that between pulmonary emphysema and pulmonary fibrosis (6).

RAS was originally proposed by the author in 2010 as a form of CLAD distinct from BOS (6). After long discussion by the pulmonary council of the International Society for Heart and Lung Transplantation (ISHLT), an updated definition of RAS was published in 2019 (1). Because chronic rejection or CLAD after lung transplantation has long been considered synonymous with BOS (2), the official introduction of RAS has changed the framework of CLAD, 
which results in the back-to-back publication of another consensus statement from ISHLT regarding CLAD in general (7).

The most important clinical characteristics of RAS are the significantly poorer outcome compared with BOS $(1,6,8)$. This fact has been reflected by the enthusiasm to investigate the new entity of CLAD at different levels using retrospective clinical data, clinical materials and animal models. Although our understanding of CLAD has increased over the last decade, why there are two distinct forms of CLAD, BOS and RAS, remains unclear. A mechanistic understanding of these two syndromes, especially how a patient develops either one of the two forms of CLAD, is an important step towards the prevention and management of CLAD.

\section{Clinical and pathological characteristics of BOS and RAS}

BOS and RAS show clearly distinct features related to their physiology, radiology, pathology and clinical outcomes (6). It is interesting to revisit the literature describing peripheral lung fibrosis resembling RAS since the beginning of clinical lung transplantation $(9,10)$. Particularly, in the initial Stanford experience of heart-lung transplantation in 1980's, interstitial fibrosis, particularly in the subpleural and septal regions, as well as pleural fibrosis, was described in addition to OB (9). Restrictive physiology with upper-lobe dominant fibrosis was also described later (11). Furthermore, in the previous definition of BOS, it was clearly stated that allograft dysfunction with restrictive physiology should be excluded from BOS (12). Thus, RAS was considered to be present throughout the history of clinical lung transplantation. The main reason this syndrome has been under-recognized and under-documented might be the definition of BOS, which primarily relies on a decline in forced expiratory volume in one second $\left(\mathrm{FEV}_{1.0}\right)$, which similarly declines in RAS. Currently, a decline in $\mathrm{FEV}_{1.0}$ is used as an indicator for CLAD development (7).

Notably, these two syndromes can convert to each other: typically, a relatively stable patient with established BOS starts to show respiratory distress with the pattern of RAS. Conversely, although rare, some established RAS patients who survive the acute exacerbation phase develop obstructive functional defects and peripheral lung hyperinflation suggestive of BOS.

In the current consensus statement from ISHLT, the definition allows for a "mixed" phenotype wherein obstructive and restrictive phenotypes coexist. Indeed, in the original study wherein we proposed RAS, we initially defined a similar mixed phenotype. However, we found the clinical features and especially the prognosis, were determined mostly by the presence or absence of the RAS phenotype. Thus, we eventually removed the mixed phenotype from the analysis and defined RAS as CLAD with restrictive physiology and BOS as CLAD without RAS (6). Further investigation of the definition and the clinical significance of the mixed phenotype is required; however, the mixed phenotype in the current definition (1) should not be confused with the phenomenon of inter-phenotype conversion as described above. Namely, some patients may develop the mixed phenotype $a b$ initio and others may overlap RAS on existing BOS, which results in a mixed phenotype.

\section{Fundamental question: why are there two forms of CLAD?}

Two remaining fundamental questions are why and how these two representative phenotypes, BOS and RAS, develop? In other words, why does the chronic "rejection" of the lung or CLAD take one of these two representative phenotypes? This may be partially explained if these two phenotypes are considered the far ends of a spectrum of a single disease entity or two distinct disease entities.

\section{Mechanisms of RAS (I): prototype of chronic lung allograft "rejection"?}

Considering putative mechanisms of RAS, the fact that multiple tissue compartments in lung allografts are involved seems important (Figure 1). It is interesting to revisit the history of experimental models of chronic rejection after lung transplantation. In mechanistic investigations of chronic rejection or BOS, reproduction of the obliterative airway disease in animal models has been of major interest because this was the disease that was thought to be the form of chronic rejection after lung transplantation (13-15). However, rodent orthotopic lung transplantation models faced challenges because the reproduction of histological patterns observed in human BOS patients was not consistent and controversial $(16,17)$. Alternatively, heterotopic tracheal transplant models have often been used to reproduce fibrotic airway obliteration despite multiple limitations $(14,15)$. Interestingly, the pattern of histological changes in previous reports on chronic lung allograft rejection using 


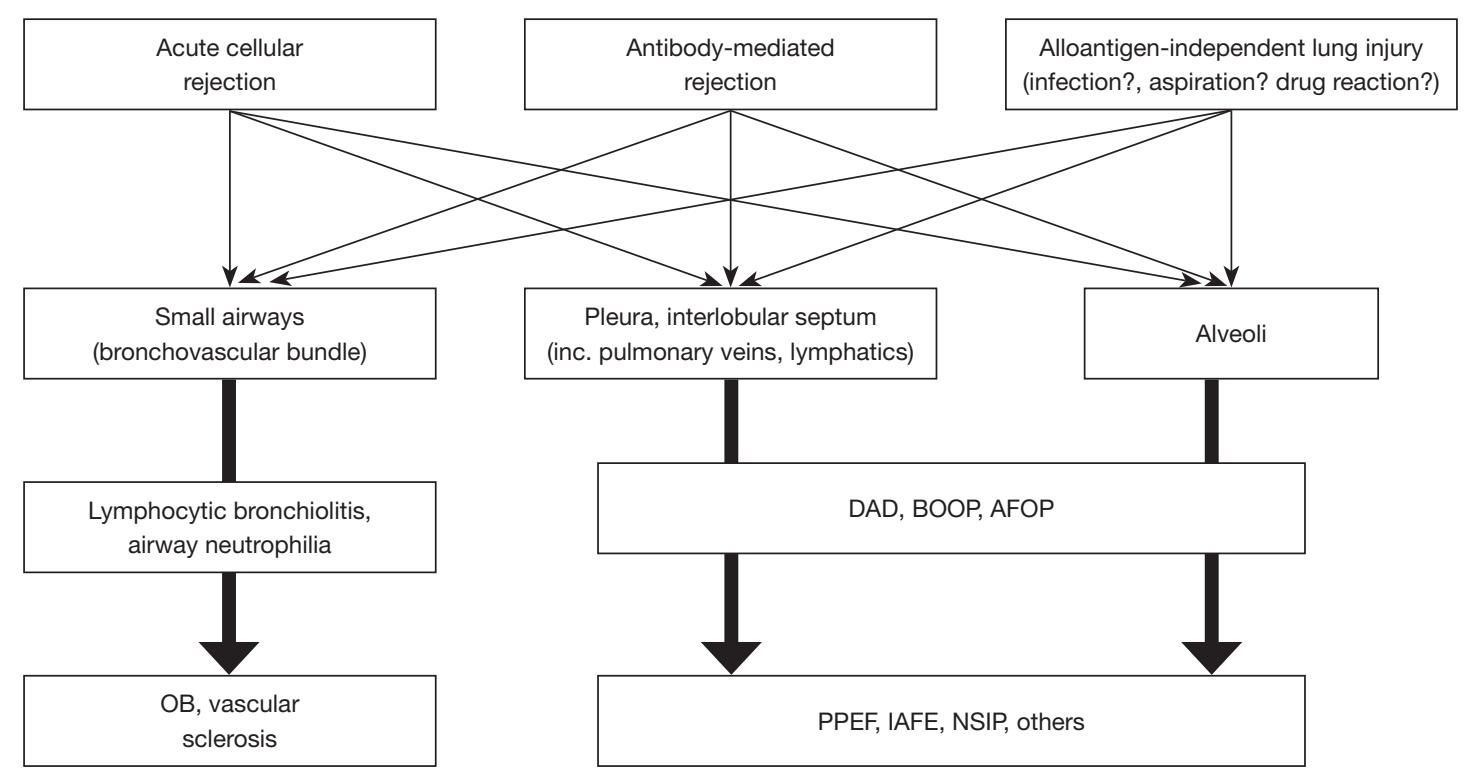

Figure 1 Putative mechanism of restrictive allograft syndrome after lung transplantation. Lung injuries including acute cellular rejection, antibody-mediated rejection, and alloantigen-independent lung injury can induce intense inflammation in different anatomical compartments of the lung. During the acute inflammatory phase, each anatomical compartment shows different pathological findings such as lymphocytic bronchiolitis and DAD, which may result in irreversible tissue remodeling and fibrosis including OB, vascular sclerosis, PPFE, IAFE, NSIP and other forms of alveolar fibrosis. OB, obliterative bronchiolitis; PPFE, pleuroparenchymal fibroelastosis; IAFE, intra-alveolar fibroelastosis; NSIP, non-specific interstitial pneumonia.

orthotopic lung transplant models have shown similarities to RAS, wherein various anatomical components including the alveoli, pleura and septum are involved in intense inflammatory and fibrotic changes $(17,18)$.

Using an orthotopic rat lung transplant model with full MHC mismatch, we applied a different duration of immunosuppression (cyclosporine) to recipient animals (19). Because fulminant immune responses with very mild immunosuppression result in RAS-like broad fibrosis in allograft lungs, we tested whether moderate immunosuppression (that is, a less intensive immune response) resulted in a milder form of RAS or a different phenotype similar to BOS (that is, fibrosis confined to airways). Indeed, we observed a different degree of RASlike broad fibrosis across different anatomical components rather than inflammation and fibrosis limited to airways as seen in BOS (19).

Similarly, in mouse orthotopic lung transplant models with minor antigen mismatch, the histological changes included pleural and septal fibrosis, peripheral lung fibrosis, fibrosis in a bronchovascular band and occasional obliterative airway disease (20-22).
Acute cellular rejection was demonstrated to be a significant risk factor for RAS (23). In a study using clinical specimens, the gene expression signature in RAS tissues was similar to rejection after kidney/liver/heart transplantation rather than BOS (24). The authors suggested that RAS should be seen as the "true" form of chronic lung allograft rejection because interstitial fibrosis is a common feature across organ rejection and is typical for RAS.

\section{Mechanisms of RAS (II): association with donor- specific antibodies (DSA)}

Furthermore, increasing evidence suggests that DSA and antibody-mediated rejection (AMR) are risk factors for RAS. A higher incidence of newly-detected human leukocyte antigen (HLA) antibodies including DSA were shown to be temporally associated with CLAD onset in RAS rather than BOS (8).

The Leuven group demonstrated that more than half of RAS patients developed DSAs while only one third of BOS patients developed DSAs, although the total number of patients with CLAD in association with DSA was larger 
in the BOS group (25). Another study demonstrated that a significant number of patients who survived AMR subsequently developed RAS (26). In that study, RAS was found exclusively in the patient group who had DSA and who experienced episodes of AMR, although such patients were only $20 \%$ of BOS patients. Interestingly, multiple studies suggested the association of RAS with DSAs against HLA class II, especially DQ (25-27), although the mechanisms involved remain to be determined.

\section{Mechanisms of RAS (III): lung injuries}

Although RAS might be induced by cellular and/or AMR, it may not explain all cases of RAS. As discussed above, one of the most prominent pathological features of RAS is its association with various patterns of lung injuries including diffuse alveolar damage (DAD) $(3,23), \mathrm{OP}(4)$ and AFOP (5). We and others have demonstrated that late new-onset DAD ( $>3$ months after lung transplantation) was significantly associated with the subsequent development of RAS, while early post-transplant DAD that is usually associated with primary graft dysfunction is not associated with RAS $(3,23)$. Progression of AFOP to RAS has also been reported $(28,29)$. Although there may be a variety of progression patterns, RAS often shows the "stair-step" pattern (30), wherein lateonset graft failure or "white out" occurs and if patients survive these episodes, they subsequently develop RAS (29).

The cause of such graft dysfunction should be examined carefully. Extensive acute cellular rejection such as A4 in the ISHLT criteria would be indistinguishable with DAD (31). Also, AMR is an important differential diagnosis and there is possibility that DSA is not detectable in the serum because of the lack of circulating DSA because of absorption by the graft or circulating DSAs other than anti-HLA antibodies. Nevertheless, despite efforts to detect these alloimmunedependent lung injuries, the results were negative in many cases and rejection was considered unlikely cause of RAS (29). Therefore, it is plausible that RAS is caused or induced by unrecognized alloimmune-independent factors.

We speculate alloantigen-independent triggers of RAS might include cytomegalovirus (CMV) or other virus infections. In the original report of RAS, CMV mismatch was shown to be a significant risk factor for RAS (6), although the result was not reproducible in other studies. Furthermore, patients with subclinical levels of CMV antigenemia and/or detection of CMV or other herpes viruses in bronchoalveolar lavage were associated with the subsequent development of RAS (unpublished data).
Increasing evidence suggests the involvement of viral infection in the development of idiopathic pulmonary fibrosis. Mechanistically, virus infection, especially by herpes virus, has been recognized as a co-factor in the progression of fibrosis $(32,33)$. A recent meta-analysis demonstrated chronic viral infections, including Epstein-Barr virus, CMV, human herpesvirus-7 and human herpesvirus- 8 , significantly increased the risk of developing idiopathic pulmonary fibrosis (34). Other than viral infection, non-specific lung injuries such as aspiration and bacterial or fungal infection might also cause acute lung injury, which in turn results in broad fibrosis of allografts as seen in RAS.

Whatever the trigger or cause of lung injury, it is evident that RAS shows a more intense inflammatory response in the lung compared with BOS. Studies using bronchoalveolar lavage fluid (BALF) demonstrated increased proinflammatory cytokines and chemokines (IL-6, IL-1R $\alpha$, CXCL10/IP-10), while VEGF was significantly reduced in RAS compared with BOS (35). The BALF of RAS patients also had higher levels of alveolar "alarmins" associated with innate immune responses including S100A9, S100A8/A9, S100A12, S100P, high-mobility group box 1 (HMGB1) (36) and pentraxin-3 (37), and proteins released from neutrophils including neutrophil elastase, $\alpha$-defensins and matrix metalloproteinase-8 (37). Interestingly, higher levels of IL-5 were also detected in the BALF of RAS patients (38), which might be associated with the high number of eosinophils in the $\operatorname{BALF}(39,40)$ and lung tissues (41) of RAS patients. Although the role of eosinophils in allograft rejection remains unclear, their involvement in humoral immune responses has been suggested (42). Furthermore, a study reported increased levels of immunoglobulins and complement proteins in the BALF of patients with RAS compared with BOS (43). These data indicate a strong association of RAS with inflammation, innate immune response and humoral immune response, the latter of which may further reinforce the association between AMR and RAS.

\section{Mechanisms of BOS: why are the airways targeted?}

Accumulating clinical and animal data suggest that BOS and RAS are distinct forms of CLAD. RAS is characterized by diffuse fibrotic processes across different anatomical compartments including the airway, pleura, septum, alveoli and vasculature, while BOS is an airway-centered disease. The next question is what causes the airway disease in BOS 


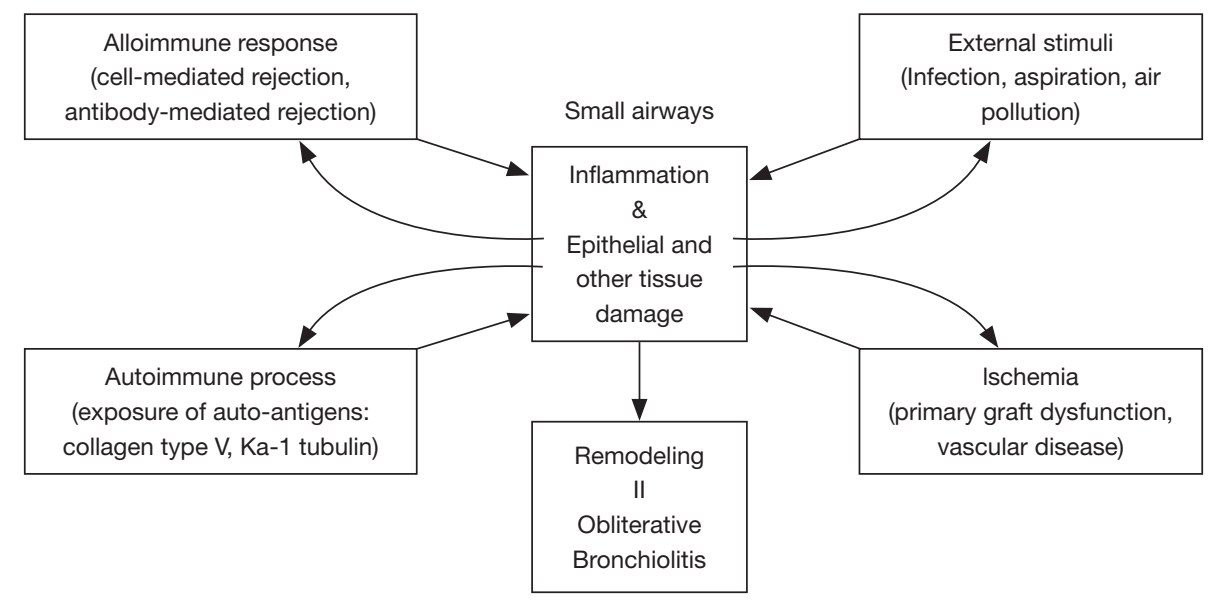

Figure 2 Putative mechanism of airway-centered inflammation and fibrosis in bronchiolitis obliterans syndrome after lung transplantation. Different injurious processes including alloimmune responses, autoimmune responses, external stimuli, and airway ischemia induces inflammation in the small airways, which results in damage to the epithelium and other tissues. These damaged tissues may further augment tissue injury (such as exposure of autoantigen, vascular damage leading to ischemia, epithelial loss resulting in reduced barrier function). Ultimately this leads to remodeling and fibrosis termed obliterative bronchiolitis, while other anatomical compartments of the lung remain relatively intact.

leaving other tissues in the allograft relatively intact? The putative mechanisms of BOS are shown in Figure 2.

\section{Mechanisms of BOS (I): acute cellular rejection and AMR}

Although there is an argument claiming that RAS may be a prototype of chronic lung allograft rejection, acute cellular and AMR is still considered a major risk factor of BOS. Relatively old literature that did not distinguish BOS and RAS reported acute cellular rejection of peripheral lung tissue (ISHLT A grade) $(44,45)$ as well as lymphocytic bronchiolitis (ISHLT B grade) $(46,47)$ were risk factors of CLAD. In the era of BOS and RAS, the role of acute cellular rejection needs reappreciation. Particularly, lymphocytic bronchiolitis was presumed to be a precursor lesion of $\mathrm{OB}$ (48), although this condition was recognized as a risk factor of RAS as well as BOS (39). It is important to note that the pathological findings of RAS include OB $(4,6)$, which indicates that small airway lesions associated with acute rejection might be common between BOS and RAS.

As discussed above, multiple reports have demonstrated that RAS has a stronger association with DSA and AMR compared with BOS (25-27). However, many patients who eventually develop BOS had also developed transient or persistent DSA with or without episodes of AMR $(25,26)$.
Taken together, acute cellular rejection and AMR are risk factors of BOS, suggesting the involvement of these alloimmune responses in the disease process of BOS. However, given the involvement of "rejection" in BOS, this may not explain why the small airways are selectively affected by the disease.

\section{Mechanisms of BOS (II): external alloantigen- independent stimuli targeting airways}

One hypothesis to explain why the disease process of $\mathrm{OB} /$ BOS is relatively selective for the airways is external stimuli targeting airways. Multiple external insults including airborne infection, aspiration and air pollution can affect lung allografts through the airways. Evidence suggests that pseudomonas colonization $(49,50)$, aspergillus colonization $(51)$, respiratory viral infection $(52,53)$ and CMV infection (54) increase the risk of CLAD. Although most evidence comes from the era before the establishment of RAS as a disease type, airborne infection is likely to increase the risk of airway-centered disease. Indeed, using a rat orthotopic lung transplant model under sufficient immunosuppression, we demonstrated that the transairway administration of lipopolysaccharide induced airway-centered inflammation and fibrosis similar to that in human BOS (55). In the present series, an excellent 
review article by Kawashima and Juvet explains the details of innate immunity leading to CLAD (56). Similarly, gastroesophageal reflux $(57,58)$ and air pollution $(59,60)$ can induce local airway-centered inflammatory processes, leading to obliterative airway fibrosis. Furthermore, we and others demonstrated that activation of an innate immune pathway through Toll-like receptor 4 directly activated fibroblasts that contributed to airway fibrosis (61).

Note that such external stimuli might affect regions beyond airways such as peripheral lung tissues. For example, aspiration might induce fulminant inflammation in the whole lung resulting in acute respiratory distress syndrome with the pathological finding of DAD. Thus, the external or alloantigen-independent factors described here are unlikely to be exclusive to BOS as a cause but could theoretically cause RAS. However, current evidence supports a hypothesis whereby airway-centered inflammation and fibrosis of OB/BOS might be partly explained by external trans-airway stimuli.

\section{Mechanisms of BOS (III): airway-specific autoantigens}

Type $\mathrm{V}$ collagen $[\operatorname{col}(\mathrm{V})]$ is a minor fibrillar collagen expressed by small airway epithelial cells and is located in the perivascular and peribronchial tissues of lungs as well as in the skin and placenta (62). Type $\mathrm{V}$ collagen is a cryptic antigen usually associated with other collagen fibers; however, it can be cleaved and exposed to the immune system by factors such as matrix metalloproteinases during intense inflammation such as ischemia reperfusion injury (63). Although $\operatorname{col}(\mathrm{V})$ is not detectable at high levels in normal lungs, elevated levels of $\operatorname{col}(\mathrm{V})$ have been reported in OB lungs in association with $\operatorname{col}(\mathrm{V})$-specific Th17 cell-mediated responses after transplantation (64).

$\mathrm{K} \alpha-1$ tubulin is also a potential autoantigen expressed by airway epithelial cells. Similar to $\operatorname{col}(\mathrm{V}), \mathrm{K} \alpha-1$ tubulin was reported to be exposed to the immune system in the context of tissue damage and the autoimmune-like response may lead to autoantibody production and OB/BOS (65).

In general, such cryptic auto-antigens might be exposed and recognized by the immune system, especially when the lung is damaged, for example, by rejection, infection, or ischemia-reperfusion injury $(62,63,66)$. Additionally, such exposure might occur even before transplantation in association with pre-transplant inflammatory diseases such as cystic fibrosis and idiopathic pulmonary fibrosis (67). Theoretically, these autoimmune-mechanisms might be associated with $\mathrm{OB}$ that is commonly observed in BOS and RAS. However, the airway-selective disease process of BOS can be preferentially explained by cellular and antibody-mediated reactions against autoantigens expressed selectively in airways such as $\mathrm{Col}(\mathrm{V})$ and $\mathrm{K} \alpha-1$ tubulin.

\section{Mechanisms of BOS (IV): ischemia and early post-transplant events}

One hypothesis to explain the airway-centered disease process of BOS is a loss of microvasculature in small airways. In observational studies using post-mortem lung allograft specimens, Luckraz et al. reported the loss of small vessels prior to the development of BOS and insufficient angioneogenesis in established BOS $(68,69)$, suggesting OB/ BOS is associated with microvascular damage around small airways. To support this hypothesis, Babu and colleagues used a murine orthotopic tracheal transplant model and demonstrated that rejecting grafts with extensive endothelial cell injury were refractory to immunotherapy, which resulted in airway fibrosis (70). Generally, ischemia or lack of oxygen or other nutritional supplies have a significant negative impact on wound healing. OB is considered a disease of tissue remodeling or failure of appropriate tissue regeneration after damage, especially in the airway epithelium (71-73). Additionally, ischemic injury may direct the airway toward further immune-mediated injury and fibrosis as discussed above. The release of DAMPs from damaged or dying cells activate innate immunity (56); the release of cryptic autoantigens might promote the autoimmune-mediated mechanisms as discussed above (62).

To attenuate the initial ischemic injury of airways and decrease the risk of subsequent airway fibrosis, bronchial artery revascularization at the time of lung transplantation is theoretically beneficial $(70,74)$. Indeed, clinical outcomes after bronchial arterial anastomosis demonstrated less central airway ischemia and related complications (75). Interestingly, there was a trend toward the delayed development of BOS in the bronchial artery revascularization group compared with the non-bronchial artery revascularization group (75). However, there are insufficient clinical data demonstrating the benefit of bronchial artery revascularization to prevent or delay the development of CLAD. This might be because ischemic or other tissue damage early after lung transplantation is not simply mediated by insufficient blood supply but by a more complex process represented by primary graft dysfunction, which is attributable to multiple peri-transplant injurious 
factors including donor lung injury related to brain death, aspiration, trauma, ventilation-induced injury, infection, cold ischemia and reperfusion injury (76). Primary graft dysfunction was demonstrated to be an important risk factor of later CLAD development $(77,78)$. Although these studies were conducted before the recognition of RAS and the phenotype of CLAD associated with primary graft dysfunction was not clarified, we demonstrated that DAD early after lung transplantation ( $<3$ months) was significantly associated with the later development of BOS, while late new-onset DAD was associated with the development of RAS (3). Early DAD is likely to be associated with early events including post-transplant ischemia-reperfusion injury and primary graft dysfunction. Interestingly, a high level of IL-6 in pre-transplant donor lung tissues was also found to be significantly associated with the later development of BOS but not RAS (79), which further supports the contention that early graft injury impacts late graft function, especially BOS. Taken together, although DAD per se is a pathological finding in alveoli that is affected by RAS rather than BOS, the early post-transplant injury detected by DAD may affect the small airways over the long-term through multiple mechanisms including ischemia, activation of innate and autoimmunity, and poor tissue regeneration, to eventually increase the risk of BOS.

\section{Lymphoid neogenesis: anatomical consideration of CLAD phenotypes}

Finally, it might be worth revisiting the issue of local chronic inflammatory responses in the context of anatomy. Both BOS and RAS are phenotypes of CLAD that share chronic inflammation and tissue remodeling as the disease process. The critical difference between these two phenotypes is the anatomical location involved in chronic inflammation and tissue remodeling: BOS is an airway-centered process while RAS involves multiple anatomical compartments including airways, pleura, septum, alveoli and vasculature. Generally, chronic inflammation often accompanies a phenomenon termed lymphoid neogenesis, wherein chronic infiltration of immune cells (such as lymphocytes) home to the tissue and interact with stromal resident cells to organize tertiary lymphoid organs similar to lymphoid follicles (80). This chronic process as is seen in infection is considered an essential protective phenomenon to confine inflammation, which could be harmful if it continues systemically (81). However, in chronic immune-mediated diseases such as autoimmune diseases, such tertiary lymphoid organs may promote chronic inflammation though various effector mechanisms including tissue destruction and antibody production (80). In organ transplantation, the organization of such lymphoid tissues has been reported in chronically rejected hearts, kidneys, livers and pancreases (82-84). We first reported lymphoid neogenesis in lung allografts using human lungs affected by CLAD and its animal models (85). However, the role of lymphoid neogenesis remains controversial or probably biphasic (86). Lymphoid neogenesis was reported in lung tissues affected by CLAD (85) and its animal models $(85,87)$ and it has also been suggested to play protective roles after lung transplantation by accommodating regulatory lymphocytes $(88,89)$. Recent studies have further revealed the detailed mechanisms whereby tertiary lymphoid tissues within transplanted organs have detrimental or protective roles (86).

Regarding the development of the different phenotypes of CLAD, lymphoid neogenesis might play a role in the progression of chronic inflammation. We examined intrapulmonary lymphoid tissues in lungs affected by CLAD and demonstrated that the distribution of lymphoid tissue in the lung affected by CLAD followed the pattern of "lymphangitic distribution", or along the lymphatics of the lung, which is also seen in other pulmonary disorders such as sarcoidosis (90). Lymphoid tissues were predominantly observed along airways or around the bronchovascular bundle, or close to the pleura and interlobular septum, which are locations affected by RAS. More recently, the histological examination of lungs affected by CLAD demonstrated intrapulmonary lymphoid tissues were more frequent in RAS than BOS (41). In animal experiments, lungs were demonstrated to mount alloimmune responses independent of secondary lymphoid organs $(87,91)$. Furthermore, our group demonstrated the local production of DSA in intrapulmonary lymphoid tissues (19). Moreover, lymphoid neogenesis in allograft lungs was demonstrated in association with Th17-mediated autoimmune responses $(62,92,93)$. Taken together, intrapulmonary lymphoid tissues may promote local cellular and humoral immune responses in detrimental cases that lead to CLAD, whereas they may also be involved in the stabilization of local immune responses. Considering the possible triggers of CLAD and complex cross-talk among the different mechanisms involved (Figure 2), lymphoid neogenesis may have an important role at the juncture of these factors in the progression of local chronic immune responses, and thus in the establishment of the different phenotypes of CLAD with the involvement of different anatomical components. 


\section{Conclusions}

There is emerging evidence to explain the mechanisms of two distinct forms of CLAD, BOS and RAS. However, these mechanisms often overlap and there is no clear explanation for the pathogenesis of each phenotype of CLAD. It is likely that these two phenotypes are multifactorial and cannot therefore be explained by a single factor or pathway. Furthermore, by definition, CLAD is a chronic disease state during which patients survive for a certain period of time along with a chronic inflammatory process. For example, even if RAS develops in a patient and is triggered by fulminant acute cellular rejection, tissue injury caused by the alloimmune response may induce autoimmune mechanisms. Because the cause-effect relationship in clinical settings is difficult to demonstrate, a simplification of the setting using animal models wherein we can test what we cannot do in clinical settings (such as significantly reducing immunosuppression in allotransplantation to examine what happens in allografts) may help. To better understand the complex mechanisms of CLAD and eventually develop effective preventive and therapeutic strategies, further investigations should focus not only on clinical samples and data but also on basic research using animal models.

\section{Acknowledgments}

We thank Edanz Group (www.edanzediting.com/ac) for editing a draft of this manuscript.

Funding: This work was funded by JSPS KAKENHI $17 \mathrm{H} 01581$.

\section{Footnote}

Provenance and Peer Review: This article was commissioned by the Guest Editors (Masaaki Sato and Dong Tian) for the series "Strategies to Achieve Long-Term Success of Lung Transplantation" published in Annals of Translational Medicine. The article was sent for external peer review organized by the Guest Editor and the editorial office.

Conflicts of Interest: The series "Strategies to Achieve LongTerm Success of Lung Transplantation" was commissioned by the editorial office without any funding or sponsorship. MS served as the unpaid Guest Editor of the series.

Ethical Statement: The author is accountable for all aspects of the work in ensuring that questions related to the accuracy or integrity of any part of the work are appropriately investigated and resolved.

Open Access Statement: This is an Open Access article distributed in accordance with the Creative Commons Attribution-NonCommercial-NoDerivs 4.0 International License (CC BY-NC-ND 4.0), which permits the noncommercial replication and distribution of the article with the strict proviso that no changes or edits are made and the original work is properly cited (including links to both the formal publication through the relevant DOI and the license). See: https://creativecommons.org/licenses/by-nc-nd/4.0/.

\section{References}

1. Glanville AR, Verleden GM, Todd JL, et al. Chronic lung allograft dysfunction: Definition and update of restrictive allograft syndrome-A consensus report from the Pulmonary Council of the ISHLT. J Heart Lung Transplant 2019;38:483-92.

2. Sato M. Chronic Lung Allograft Dysfunction after Lung Transplantation: the Moving Target. Gen Thorac Cardiovasc Surg 2013;61:67-78.

3. Sato M, Hwang DM, Ohmori-Matsuda K, et al. Revisiting the pathologic finding of diffuse alveolar damage after lung transplantation. J Heart Lung Transplant 2012;31:354-63.

4. Ofek E, Sato M, Saito T, et al. Restrictive allograft syndrome post lung transplantation is characterized by pleuroparenchymal fibroelastosis. Mod Pathol 2013;26:350-6.

5. Paraskeva M, McLean C, Ellis S, et al. Acute fibrinoid organizing pneumonia after lung transplantation. Am J Respir Crit Care Med 2013;187:1360-8.

6. Sato M, Waddell TK, Wagnetz U, et al. Restrictive Allograft Syndrome (RAS): a novel form of chronic allograft dysfunction after lung transplantation. J Heart Lung Transplant 2011;30:735-42.

7. Verleden GM, Glanville AR, Lease ED, et al. Chronic lung allograft dysfunction: Definition, diagnostic criteria, and approaches to treatment-A consensus report from the Pulmonary Council of the ISHLT. J Heart Lung Transplant 2019;38:493-503.

8. Todd JL, Jain R, Pavlisko EN, et al. Impact of forced vital capacity loss on survival after the onset of chronic lung allograft dysfunction. Am J Respir Crit Care Med 2014;189:159-66. 
9. Yousem SA, Burke CM, Billingham ME. Pathologic pulmonary alterations in long-term human heart-lung transplantation. Hum Pathol 1985;16:911-23.

10. Martinu T, Howell DN, Davis RD, et al. Pathologic correlates of bronchiolitis obliterans syndrome in pulmonary retransplant recipients. Chest 2006;129:1016-23.

11. Pakhale SS, Hadjiliadis D, Howell DN, et al. Upper lobe fibrosis: a novel manifestation of chronic allograft dysfunction in lung transplantation. J Heart Lung Transplant 2005;24:1260-8.

12. Estenne M, Maurer JR, Boehler A, et al. Bronchiolitis obliterans syndrome 2001: an update of the diagnostic criteria. J Heart Lung Transplant 2002;21:297-310.

13. Kuo E, Bharat A, Dharmarajan S, et al. Animal models for bronchiolitis obliterans syndrome following human lung transplantation. Immunol Res 2005;33:69-81.

14. Sato M, Keshavjee S, Liu M. Translational Research: Animal Models of Obliterative Bronchiolitis after Lung Transplantation. Am J Transplant 2009;9:1981-7.

15. Jungraithmayr W, Jang JH, Schrepfer S, et al. Small animal models of experimental obliterative bronchiolitis. Am J Respir Cell Mol Biol 2013;48:675-84.

16. Hirschburger M, Greschus S, Kuchenbuch T, et al. Lung transplantation in the Fischer 344-->Wistar Kyoto rat strain combination is not suitable to study bronchiolitis obliterans. J Heart Lung Transplant 2007;26:390-8.

17. Matsumura Y, Marchevsky A, Zuo XJ, et al. Assessment of pathological changes associated with chronic allograft rejection and tolerance in two experimental models of rat lung transplantation. Transplantation 1995;59:1509-17.

18. Fan L, Benson HL, Vittal R, et al. Neutralizing IL-17 prevents obliterative bronchiolitis in murine orthotopic lung transplantation. Am J Transplant 2011;11:911-22.

19. Miyamoto E, Motoyama H, Sato M, et al. Association of Local Intrapulmonary Production of Antibodies Specific to Donor Major Histocompatibility Complex Class I With the Progression of Chronic Rejection of Lung Allografts. Transplantation 2017;101:e156-65.

20. Vanaudenaerde B, Verleden S, Neyrinck A, et al. A New Step in the Marathon of Understanding Chronic Rejection after Lung Transplantation. Am J Respir Cell Mol Biol 2017;56:683-4.

21. Martinu T, Oishi H, Juvet SC, et al. Spectrum of chronic lung allograft pathology in a mouse minor-mismatched orthotopic lung transplant model. Am J Transplant 2019;19:247-58.
22. Yamada Y, Windirsch K, Dubs L, et al. Chronic Airway Fibrosis in Orthotopic Mouse Lung Transplantation Models-An Experimental Reappraisal. Transplantation 2018;102:e49-58.

23. Shino MY, Weigt SS, Li N, et al. CXCR3 ligands are associated with the continuum of diffuse alveolar damage to chronic lung allograft dysfunction. Am J Respir Crit Care Med 2013;188:1117-25.

24. Sacreas A, Yang JYC, Vanaudenaerde BM, et al. The common rejection module in chronic rejection post lung transplantation. PLoS One 2018;13:e0205107.

25. Verleden SE, Vanaudenaerde BM, Emonds MP, et al. Donor-specific and -nonspecific HLA antibodies and outcome post lung transplantation. Eur Respir J 2017. doi: 10.1183/13993003.01248-2017.

26. Roux A, Bendib Le Lan I, Holifanjaniaina S, et al. Antibody-Mediated Rejection in Lung Transplantation: Clinical Outcomes and Donor-Specific Antibody Characteristics. Am J Transplant 2016;16:1216-28.

27. Walton DC, Hiho SJ, Cantwell LS, et al. HLA Matching at the Eplet Level Protects Against Chronic Lung Allograft Dysfunction. Am J Transplant 2016;16:2695-703.

28. Costa AN, Carraro RM, Nascimento EC, et al. Acute Fibrinoid Organizing Pneumonia in Lung Transplant: The Most Feared Allograft Dysfunction. Transplantation 2016;100:e11-2.

29. Verleden SE, Gottlieb J, Dubbeldam A, et al. "WhiteOut" After Lung Transplantation: A Multicenter Cohort Description of Late Acute Graft Failure. Am J Transplant 2017;17:1905-11.

30. Sato M, Hwang DM, Waddell TK, et al. Progression pattern of restrictive allograft syndrome after lung transplantation. J Heart Lung Transplant 2013;32:23-30.

31. Stewart S, Fishbein MC, Snell GI, et al. Revision of the 1996 working formulation for the standardization of nomenclature in the diagnosis of lung rejection. J Heart Lung Transplant 2007;26:1229-42.

32. Vannella KM, Moore BB. Viruses as co-factors for the initiation or exacerbation of lung fibrosis. Fibrogenesis Tissue Repair 2008;1:2.

33. Naik PK, Moore BB. Viral infection and aging as cofactors for the development of pulmonary fibrosis. Expert Rev Respir Med 2010;4:759-71.

34. Sheng G, Chen P, Wei Y, et al. Viral Infection Increases the Risk of Idiopathic Pulmonary Fibrosis: A MetaAnalysis. Chest 2019. [Epub ahead of print].

35. Verleden SE, Ruttens D, Vos R, et al. Differential cytokine, 
chemokine and growth factor expression in phenotypes of chronic lung allograft dysfunction. Transplantation 2015;99:86-93.

36. Saito T, Liu M, Binnie M, et al. Distinct expression patterns of alveolar "alarmins" in subtypes of chronic lung allograft dysfunction. Am J Transplant 2014;14:1425-32.

37. Saito T, Liu M, Binnie M, et al. Upregulation of alveolar neutrophil enzymes and long pentraxin-3 in human chronic lung allograft dysfunction subtypes. J Thorac Cardiovasc Surg 2018;155:2774-6.e2.

38. Berastegui C, Gomez-Olles S, Sanchez-Vidaurre S, et al. BALF cytokines in different phenotypes of chronic lung allograft dysfunction in lung transplant patients. Clin Transplant 2017. doi: 10.1111/ctr.12898.

39. Verleden SE, Ruttens D, Vandermeulen E, et al. Bronchiolitis obliterans syndrome and restrictive allograft syndrome: do risk factors differ? Transplantation 2013;95:1167-72.

40. Verleden SE, Ruttens D, Vandermeulen E, et al. Elevated bronchoalveolar lavage eosinophilia correlates with poor outcome after lung transplantation. Transplantation 2014;97:83-9.

41. Vandermeulen E, Lammertyn E, Verleden SE, et al. Immunological diversity in phenotypes of chronic lung allograft dysfunction: a comprehensive immunohistochemical analysis. Transpl Int 2017;30:134-43.

42. Goldman M, Moine AL, Braun M, et al. A role for eosinophils in transplant rejection. Trends Immunol 2001;22:247-51.

43. Vandermeulen E, Verleden SE, Bellon H, et al. Humoral immunity in phenotypes of chronic lung allograft dysfunction: A broncho-alveolar lavage fluid analysis. Transpl Immunol 2016;38:27-32.

44. Heng D, Sharples LD, McNeil K, et al. Bronchiolitis obliterans syndrome: incidence, natural history, prognosis, and risk factors. J Heart Lung Transplant 1998;17:1255-63.

45. Hachem RR, Khalifah AP, Chakinala MM, et al. The significance of a single episode of minimal acute rejection after lung transplantation. Transplantation 2005;80:1406-13.

46. Husain AN, Siddiqui MT, Holmes EW, et al. Analysis of risk factors for the development of bronchiolitis obliterans syndrome. Am J Respir Crit Care Med 1999;159:829-33.

47. Glanville AR, Aboyoun CL, Havryk A, et al. Severity of lymphocytic bronchiolitis predicts long-term outcome after lung transplantation. Am J Respir Crit Care Med
2008;177:1033-40.

48. Yousem SA. Lymphocytic bronchitis/bronchiolitis in lung allograft recipients. Am J Surg Pathol 1993;17:491-6.

49. Botha P, Archer L, Anderson RL, et al. Pseudomonas aeruginosa colonization of the allograft after lung transplantation and the risk of bronchiolitis obliterans syndrome. Transplantation 2008;85:771-4.

50. Gregson AL, Wang X, Weigt SS, et al. Interaction between Pseudomonas and CXC chemokines increases risk of bronchiolitis obliterans syndrome and death in lung transplantation. Am J Respir Crit Care Med 2013;187:518-26.

51. Weigt SS, Elashoff RM, Huang C, et al. Aspergillus colonization of the lung allograft is a risk factor for bronchiolitis obliterans syndrome. Am J Transplant 2009;9:1903-11.

52. Kumar D, Erdman D, Keshavjee S, et al. Clinical impact of community-acquired respiratory viruses on bronchiolitis obliterans after lung transplant. Am J Transplant 2005;5:2031-6.

53. Allyn PR, Duffy EL, Humphries RM, et al. Graft Loss and CLAD-Onset Is Hastened by Viral Pneumonia After Lung Transplantation. Transplantation 2016;100:2424-31.

54. Paraskeva M, Bailey M, Levvey BJ, et al. Cytomegalovirus replication within the lung allograft is associated with bronchiolitis obliterans syndrome. Am J Transplant 2011;11:2190-6.

55. Takahagi A, Sato M, Chen-Yoshikawa TF, et al. LPSinduced airway-centered inflammation leading to BOS-like airway remodeling distinct from RAS-like fibrosis in rat lung transplantation. Transplantation 2020. [Epub ahead of print].

56. Kawashima M, Juvet SC. The Role of Innate Immunity in the Long-term Outcome of Lung Transplantation. Ann Transl Med 2020. [Epub ahead of print].

57. Hartwig MG, Anderson DJ, Onaitis MW, et al. Fundoplication after lung transplantation prevents the allograft dysfunction associated with reflux. Ann Thorac Surg 2011;92:462-8; discussion 468-9.

58. D'Ovidio F, Mura M, Ridsdale R, et al. The effect of reflux and bile acid aspiration on the lung allograft and its surfactant and innate immunity molecules SP-A and SP-D. Am J Transplant 2006;6:1930-8.

59. Bhinder S, Chen H, Sato M, et al. Air pollution and the development of posttransplant chronic lung allograft dysfunction. Am J Transplant 2014;14:2749-57.

60. Nawrot TS, Vos R, Jacobs L, et al. The impact of traffic 
air pollution on bronchiolitis obliterans syndrome and mortality after lung transplantation. Thorax 2011;66:748-54.

61. Kawashima M, Sato M, Murakawa T, et al. Role of Tolllike Receptor 4 Expressed by Fibroblasts in Allograft Fibrosis in Mouse Orthotopic Tracheal Transplantation. Transplant Proc 2018;50:3863-72.

62. Weber DJ, Wilkes DS. The role of autoimmunity in obliterative bronchiolitis after lung transplantation. Am J Physiol Lung Cell Mol Physiol 2013;304:L307-11.

63. Iwata T, Chiyo M, Yoshida S, et al. Lung transplant ischemia reperfusion injury: metalloprotease inhibition down-regulates exposure of type $V$ collagen, growthrelated oncogene-induced neutrophil chemotaxis, and tumor necrosis factor-alpha expression. Transplantation 2008;85:417-26.

64. Burlingham WJ, Love RB, Jankowska-Gan E, et al. IL17-dependent cellular immunity to collagen type $\mathrm{V}$ predisposes to obliterative bronchiolitis in human lung transplants. J Clin Invest 2007;117:3498-506.

65. Goers TA, Ramachandran S, Aloush A, et al. De Novo Production of K-\{alpha\}1 Tubulin-Specific Antibodies: Role in Chronic Lung Allograft Rejection. J Immunol 2008;180:4487-94.

66. Fukami N, Ramachandran S, Saini D, et al. Antibodies to MHC class I induce autoimmunity: role in the pathogenesis of chronic rejection. J Immunol 2009; 182:309-18.

67. Tiriveedhi V, Gautam B, Sarma NJ, et al. Pre-transplant antibodies to Kalpha1 tubulin and collagen- $\mathrm{V}$ in lung transplantation: clinical correlations. J Heart Lung Transplant 2013;32:807-14.

68. Luckraz H, Goddard M, McNeil K, et al. Microvascular changes in small airways predispose to obliterative bronchiolitis after lung transplantation. J Heart Lung Transplant 2004;23:527-31.

69. Luckraz H, Goddard M, McNeil K, et al. Is obliterative bronchiolitis in lung transplantation associated with microvascular damage to small airways? Ann Thorac Surg 2006;82:1212-8.

70. Babu AN, Murakawa T, Thurman JM, et al. Microvascular destruction identifies murine allografts that cannot be rescued from airway fibrosis. J Clin Invest 2007;117:3774-85.

71. Adams BF, Brazelton T, Berry GJ, et al. The role of respiratory epithelium in a rat model of obliterative airway disease. Transplantation 2000;69:661-4.
72. Sato M, Keshavjee S. Bronchiolitis obliterans syndrome: alloimmune-dependent and -independent injury with aberrant tissue remodeling. Semin Thorac Cardiovasc Surg 2008;20:173-82.

73. Sato M, Liu M, Anraku M, et al. Allograft airway fibrosis in the pulmonary milieu: a disorder of tissue remodeling. Am J Transplant 2008;8:517-28.

74. Shigemura N, Tane S, Noda K. The Bronchial Arterial Circulation in Lung Transplantation: Bedside to Bench to Bedside, and Beyond. Transplantation 2018;102:1240-9.

75. Pettersson GB, Karam K, Thuita L, et al. Comparative study of bronchial artery revascularization in lung transplantation. J Thorac Cardiovasc Surg 2013;146:894-900.e3.

76. de Perrot M, Liu M, Waddell TK, et al. Ischemiareperfusion-induced lung injury. Am J Respir Crit Care Med 2003;167:490-511.

77. Fiser SM, Tribble CG, Long SM, et al. Ischemiareperfusion injury after lung transplantation increases risk of late bronchiolitis obliterans syndrome. Ann Thorac Surg 2002;73:1041-7; discussion 1047-8.

78. Daud SA, Yusen RD, Meyers BF, et al. Impact of immediate primary lung allograft dysfunction on bronchiolitis obliterans syndrome. Am J Respir Crit Care Med 2007;175:507-13.

79. Saito T, Takahashi H, Kaneda H, et al. Impact of cytokine expression in the pre-implanted donor lung on the development of chronic lung allograft dysfunction subtypes. Am J Transplant 2013;13:3192-201.

80. Aloisi F, Pujol-Borrell R. Lymphoid neogenesis in chronic inflammatory diseases. Nat Rev Immunol 2006;6:205-17.

81. Moyron-Quiroz JE, Rangel-Moreno J, Kusser K, et al. Role of inducible bronchus associated lymphoid tissue (iBALT) in respiratory immunity. Nat Med 2004;10:927-34.

82. Thaunat O, Patey N, Morelon E, et al. Lymphoid neogenesis in chronic rejection: the murderer is in the house. Curr Opin Immunol 2006;18:576-9.

83. Thaunat O, Field AC, Dai J, et al. Lymphoid neogenesis in chronic rejection: evidence for a local humoral alloimmune response. Proc Natl Acad Sci U S A 2005;102:14723-8.

84. Baddoura FK, Nasr IW, Wrobel B, et al. Lymphoid neogenesis in murine cardiac allografts undergoing chronic rejection. Am J Transplant 2005;5:510-6.

85. Sato M, Hirayama S, Hwang DM, et al. The role of intrapulmonary de novo lymphoid tissue in obliterative bronchiolitis after lung transplantation. J Immunol 2009;182:7307-16. 
86. Koenig A, Thaunat O. Lymphoid Neogenesis and Tertiary Lymphoid Organs in Transplanted Organs. Front Immunol 2016;7:646.

87. Wagnetz D, Sato M, Hirayama S, et al. Rejection of tracheal allograft by intrapulmonary lymphoid neogenesis in the absence of secondary lymphoid organs. Transplantation 2012;93:1212-20.

88. Li W, Bribriesco AC, Nava RG, et al. Lung transplant acceptance is facilitated by early events in the graft and is associated with lymphoid neogenesis. Mucosal Immunol 2012;5:544-54.

89. Li W, Gauthier JM, Higashikubo R, et al. Bronchusassociated lymphoid tissue-resident Foxp3+ T lymphocytes prevent antibody-mediated lung rejection. J Clin Invest

Cite this article as: Sato M. Bronchiolitis obliterans syndrome and restrictive allograft syndrome after lung transplantation: why are there two distinct forms of chronic lung allograft dysfunction? Ann Transl Med 2020;8(6):418. doi: 10.21037/ atm.2020.02.159
2019;129:556-68.

90. Sato M, Hirayama S, Matsuda Y, et al. Stromal activation and formation of lymphoid-like stroma in chronic lung allograft dysfunction. Transplantation 2011;91:1398-405.

91. Gelman AE, Li W, Richardson SB, et al. Cutting edge: Acute lung allograft rejection is independent of secondary lymphoid organs. J Immunol 2009;182:3969-73.

92. Deteix C, Attuil-Audenis V, Duthey A, et al. Intragraft Th17 infiltrate promotes lymphoid neogenesis and hastens clinical chronic rejection. J Immunol 2010;184:5344-51.

93. Grogan JL, Ouyang W. A role for Th17 cells in the regulation of tertiary lymphoid follicles. Eur J Immunol 2012;42:2255-62. 